sein Relief erst, wenn man es im Zusammenhalt mit den übrigen besitzrechtlichen Arbeiten des Verfassers liest.

Actio e interdictum befakt sich mit dem Nachweis, daf wo in den Digesten ein klassisches Interdikt, "actio" genannt wird, was nicht selten ist, das allemal Interpolation ist. Die "Note critiche" endlich suchen den bekannten Widerspruch zwischen D. 28, 7, 25 (wonach der servus heres iustitutus sine iussa domini condicioni parere non potest) und $35,1,5,1$, wonach er es allerdings kann, durch Interpolationsnachweis zu erklären.

Alle die besprochenen Schriften - ich bedauere lebhaft nicht mehr Raum zu ausführlicheren Darlegungen zu finden - zeugen von gewissenhaftester Forschung und weitem Blick und mögen Jedermann zu ernstlichem Studium empfohlen werden.

Gries-Bozen November 1912.

Mitteis.

\title{
Nachtrags miszelle.
}

[Nene Urkunden.] Das abgelaufene Jahr hat neuerdings eine gröfere Anzahl von Papyruspublikationen gebracht. Über einige derselben wird von H. Lewald und J. Partsch speziell referiert werden; deshalb kann ich mich begnügen, den interessanten Band der Publicazioni della soc. Italiana per la ricerca dei Pap. gr. e lat. in Egitto (Pap. Greci e Latini Vol. 1) sowie Jean Maspéros Fortsetzung der Edition der Apbrodite-Papyri und Gradenwitz-Preisigke-Spiegelberg, ein Erbstreit aus dem ptolemäischen Ägypten, Straß̧burg, Trübner 1912 (Schriften der Wiss. Ges. zu Strafburg 13. Heft) hier nur zu nennen. Nicht die gleiche Enthaltsamkeit kanv ich bezüglich des von Hunt herausgegebenen neuesten Bandes der Oxyrhynchospapyri üben, weil dieser neue Tatsachen für einige von mir schon wiederholt besprochene Fragen beibringt, zu welchen ich Stellung nehmen möchte.

I. Der neue Oxyrhynchosband, wieder ein Zeichen der erstaunlichen Leistungsfähigkeit seines Verfassers, bringt zunächst in den Nummern 1166-1184 eine Anzahl theologischer und literarischer Texte, teilweise von höchstem Interesse, über welche jedoch die Fachleute zu sprechen hahen. Sodann gibt Nr. 1186, aus dem 4. Jahrhundert, das Edikt eines Praeses Thebaidis, uamens Aurelius Herodes, welches die Anwendung

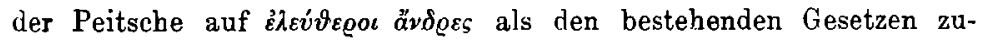
widerlaufend rügt, da dieses Werkzeug selbst gegenüber Sklaven unpassend, wenn auch nicht schlechthin verboten sei. Die Regel selbst ist bekannt und durch juristische wie historische Quellen bezeugt; dak sie in der Praxis gelegentlich verletzt wurde, ist für Ägypten am wenigsten verwunderlich. Leider sagt das Edikt nicht, auf welche 
Kategorien freier Personen man die Peitsche angewendet hat; für die Fellachen wären sie ein altäberkommenes Privilegium odiosum gewesen. Da die $\dot{\varepsilon} \lambda \varepsilon v v_{\varepsilon} \varrho \circ$ ganz allgemein als von ihr eximiert bezeichnet werden, sieht man, daßs wenigstens in dieser Beziehung die Dediticier, denn das sind die Fellachen, keine besondere Stellung einnahmen. Ieider ist das Hauptwort, welches die Peitsche bezeichnet, nicht mit

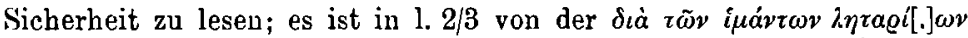

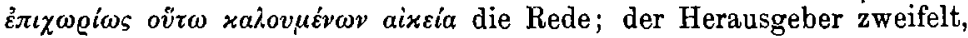

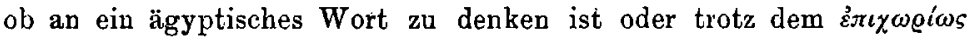

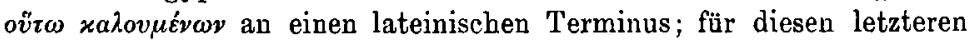
Fall denkt er an lethalium. Da aber, wie er mitteilt, das zweifelbafte $\tau$ auch für $\gamma$ gelesen werdeu kanu, möchte ich eine Gräzisierung von ligaria vermuten, welches nacb Ducange zusammengebundene Sehnen bedeuten kann; auch die buneura in Lips. 40 III 20 sind wohl Riemen, die der gründlicheren Wirkung wegen verflochten gewesen sein werden.

Über das eidliche Gestellungsversprechen Nr. 1195 hat bereits Wenger oben S. 489 f. dieses Bandes gehandelt.

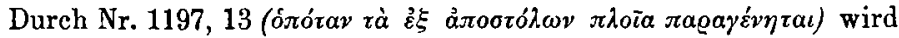
die Deutung gestützt, welche Wilcken Chrestomathie 1, Finleit. zu Nr. 443 den áróorolot in Amb. 138, 10 cf. Lond. 256, 9/10 gegeben hat; es scheinen die von der Zentralstelle ausgeheuden Aufträge (zur Einhebung der $\dot{z} \mu \beta o \lambda \dot{\eta}$, u. ä.) zu sein.

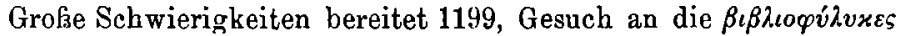

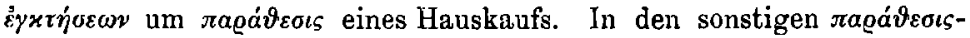

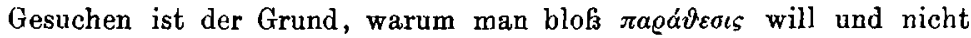

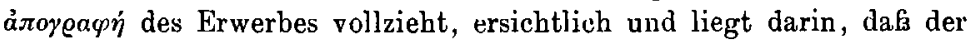

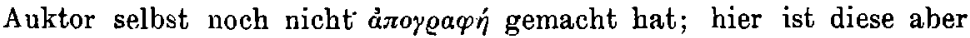
erfolgt und so verstehen wir nicht, warum die Käuferin ihr Begehren

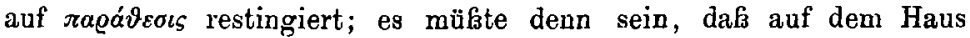
wegen Verpfändung ein Veräuferungsverbot lag o. ä., ohne dafs dies gesagt ist. Zu bemerken ist übrigens, daß der Papyrus unvollständig erhalten ist; es bleibt also die Möglichkeit, daf3 der Grund im Gesuch genannt war, aus dem von ảroy@aø $\eta$ abgesehen wurde.

Eine für die Lehre von der Behandlung der Cheirographa instruktive Urkunde - es handelt sich dabei um einen chirographarischen Kaufvertrag - enthält Nr. 1200 vom J. 266. Sie zeichnet sich dadurch aus, das nach dem Gesuch um $\delta \eta \mu \sigma o i \omega \sigma$ s die Bitte um Mitteilung

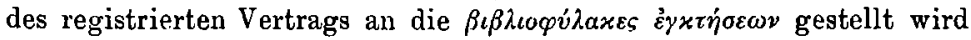
und dazu noch die Erledigung des an den $\dot{\alpha} \varrho \iota \delta \iota x \alpha \sigma \tau \eta^{\prime} s$ gerichteten

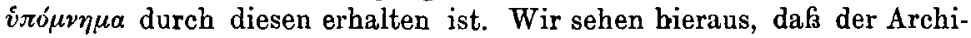
dikastes sich auch zu dieser Vermittlung bereitfinden lieb; er schreibt:

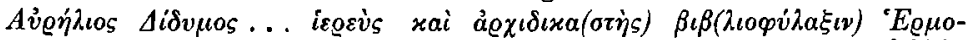

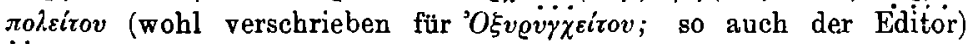

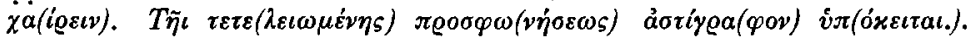

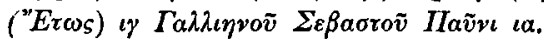

Das Wort $\pi$ oo graphums ist schon aus P. Grenf. 2, 701.20 und 71 II 1.27 bekannt, 
bezieht sich aber dort auf die Nachtragsregistrierung des Chirographum, während diese hier wie stets, wo sie beim $\dot{a} e x \iota \delta \iota \varkappa \alpha \sigma \tau \eta \dot{s}$ statt-

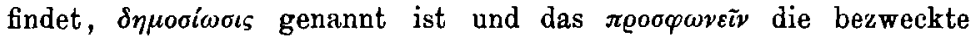

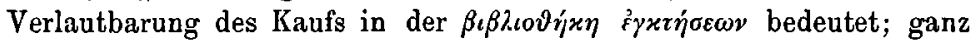

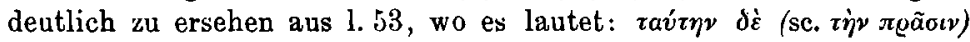

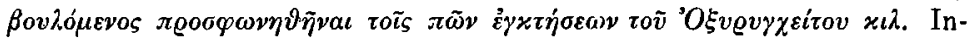
dessen erklärt sich die zweifache Verwendung des Wortes leicht, wenn man es, wie hier geschehen, mit "verlautbaren" übersetzt. - In l. 51 wird insbesondere noch betont, dafe die $\delta \eta \mu o \sigma i \omega \sigma \iota s$ deswegen anstands-

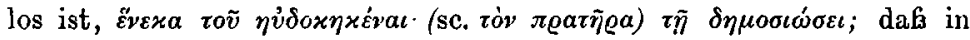
einzelnen Chirographa (vgl. auch in dem unsern, lin. 51) auch wirklich

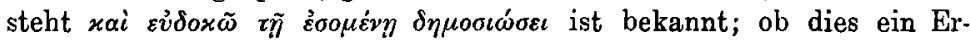
fordernis für die Registrierung war und der Destinatar eines KaufCheirographon nicht ohne weiteres es einseitig registrieren lassen konnte, steht für mich noch dahin. - Der Papyrus entscheidet im bejahenden Sinne auch die Frage, ob ein registriertes xęoóyeaqov

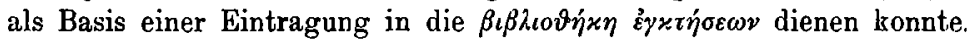
Denn jedenfalls ist solche in unserem Fall bezweckt.

Wichtiger noch als dieser ist in meinen Augen der Papyrus Nr. 1208:

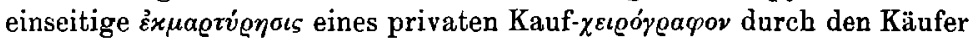

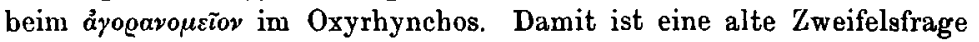
im bejahenden Sinne gelöst, nänlich die, ob die Nachtragsregistrierung

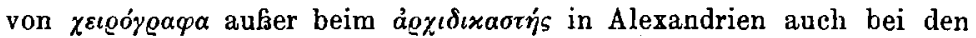
Agoranomen in der $\chi \dot{\omega} \varrho \alpha$ erfolgen konnte. Die Frage hatten wir uns schon seit langem gestellt; zuletzt hatte ich selbst sie in den Grund. zügen der Papyruskunde 2, 86 neuerlich erörtert. Manches schien zu ihrer Bejahung hinzudrängen. So vor allem der in den Papyri vorkommende

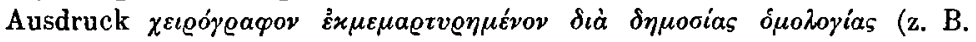
BGU. 619, 14-16, dazu Eger, Grundbuchwesen 95, A. 8 und Oxy. 1199, 19;

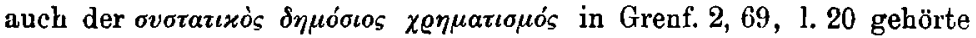
auf dieses Blatt. Es schien naheliegend, diese einfache und bequeme Art der Nachtragsregistrierung als möglich anzusehen; wenn man auch ich selbst - an sie bisher nicht hat glauben wollen, so trifft uns nicht der Vorwurf eines Ubersebens, da wir uns vielmehr mit dem Zweifel genügend gequält hatten. Vielmehr hatten wir uns gesagt: Wozu die umständliche Prozedur der eine Reise nach Alexandrien erfordernden $\delta \eta \mu \sigma o i \omega \sigma i s$ vor dem Erzrichter, wenn man denselben Erfolg zu Hause erreichen konnte? So hatte ich denn, Röm. Priv. R. 1,

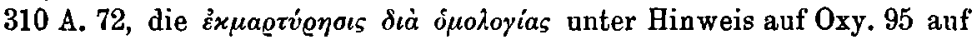
eine von beiden Parteien einverständlich vollzogene Reproduktion des chirographischen Vertrags vor dem Agoranomen gedeutet. Nun zeigt es sich wieder, wie trügerisch das Papyrusmaterial sein kann und wie sehr man sich büten mußs, aus dem vorhandenen immer nur durch den Zufall begrenzten Fundmaterial ein Argumentum a Silentio zu ziehen; mag dasselbe auch noch so sehr durch innere Erwägungen unterstützt erscheinen. Das mögen sich diejenigen gesagt sein lassen, welche wie J. Kohler über die zahlreichen in der Papyrus-Chrestomathie 
offengelassenen Fragen ihr Erstaunen äuGern; nur der dieses Gebiets Unkundige sucht hier sofort nach festen Resultaten und ist enttäuscht, wenn ihm statt solcher $\mathrm{Zweifel}$ entgegentreten; wer in vieljähriger A.rbeit es gelernt hat mit der unendlichen Lückenhaftigkeit dieser Überlieferung zu rechuen, der weiß zu warten, weil er weiß, wie sehr hier mit Überraschungen zu rechnen ist und wie wenig es angeht, die „toten Strecken ${ }^{4}$ der Überlieferung mit hypothetischen Konstruktionen auszufüllen. Nun entstehen natürlich neue Fragen: Warum hat man

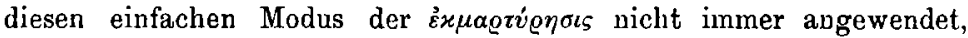
vielmehr so oft sich mit der umständlichen $\delta \eta \mu o \sigma i \omega \sigma \iota s$ in Alexandrien gequält? Das muf seinen Grund haben in bestimmten Vorschriften, welche die $\delta \eta \mu \sigma i ́ \omega \sigma \iota s$ beim Archidikastes für gewisse Arten von zвı@óreapa obligatorisch erklärten; man hat denn auch schon versucht nachzuweisen, dafs die alexandrinische Registrierung gewisse Be-

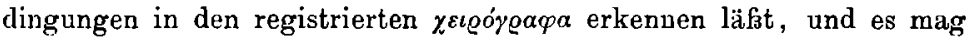
wohl sein, dafs dem so war; aber über Vermutungen ist derzeit nicht hinauszukommen.

Merkwürdig ist ferner: als Verkäufer wird genannt ein unter väterlicher Gewalt ( $(i \pi \dot{0} \tau \tilde{\eta} \chi \varepsilon \varepsilon \varrho i$ lin. 6) stehender filius familias; er verkauft ein von seiner Mutter ererbtes $1 / 5$ Anteil gewisser Grundstücke

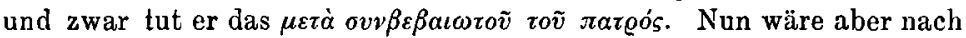
römischem Recht, das auf diese Parteien (Aurelioi, a 0 291) Anwendong fand, das Haus Ejgentum des Vaters gewesen jure potestatis; wir finden hier eine neue Äufserung des Volksrechts, welches das Eigentum an den bona materna schon vor Constantin dem Kind zuschreibt und den Vater nur zu dessen Verüuferung die Zustimmung ( $\sigma v v \beta \varepsilon$ $\beta a \iota \omega r \eta \dot{s}$ ) geben läfst. Man beachte endlich auch die hier stattfindende Stellvertretung; die Käuferin ist vertreten durch ihren Vater, welcher für sie den Kauf stipuliert.

1201: Agnition und Erteilung einer bon. possessio intestati1) (vgl. P. Giss. publiz, v. Eger, diese Ztschr. 32, 378f.). Zu Nr. 1203 verweise ich auf die Besprechung dieses Stückes in der Anzeige von Lewald. 1204 enthält eine verwaltungsrechtliche Eingabe an den Strategen, von einer Person, welche ungehörigerweise zum Dezemprinat nominiert worden zu sein behauptet. In die Eingabe eingeflochten ist die Abschrift eines Verhandlungsprotokolls, aufgenommen beim xa७olıxós

1) Denn diese ist offeubar gemeint mit den Worten 1.6 airov́ $\mu \varepsilon v o s$

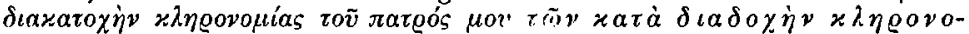

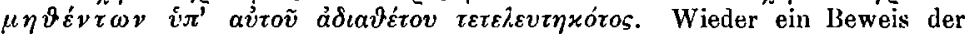
von mir schon RPR. 1, 104 A. 28 verteidigten Lehre von $R$. de Ruggiero,

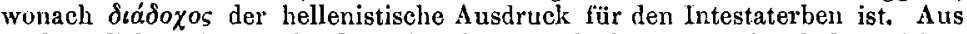
nachträglichem Material, das ich seither noch dazu gefunden habe, zitiere

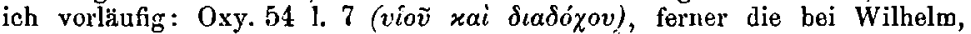
Beitr. z. griech. Jnschriftenkunde p. $192 \mathrm{f}$. u. Nachtr. p. 317 angeführte In-

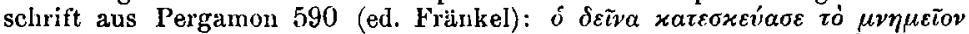

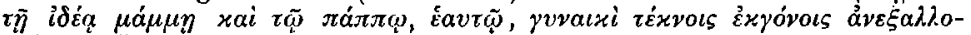
$\tau \varrho i \omega \tau o v \tilde{\varepsilon} \omega \varsigma \delta \iota \alpha \delta \circ \chi \tilde{\eta} \varsigma$, d. h. wohl unveräußerlich, solange noch Nachkommenschaft da ist. 
(Rationalis), welcher zunächst fragt, ob die Einspruchsfrist nicht versäumt ist und dann, da der Nominant nicht anwesend ist, befiehlt, daß diesem eine $\pi$ a $a \gamma \gamma \varepsilon \lambda i a$ zugestellt werde; vielleicht ist der vorliegende (nicht vollständig erhaltene) Papyrus gerade das vлó $\mu \nu \eta \mu \alpha$, in wisem der Strateg um diese Zustellung gebeten wird. Der Hergang erinnert stark an die in den Vat. Fr. enthaltenen Bestimmungen über dic Dcnunciatio. Bemerkenswert und neu ist: das Protokoll schliefit mit den

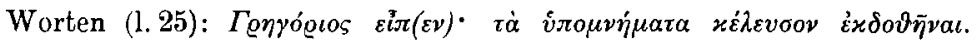

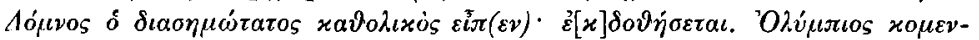

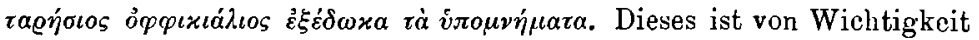

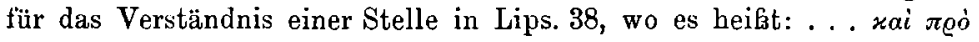

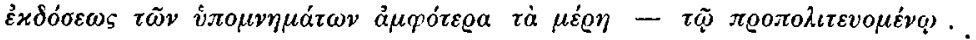

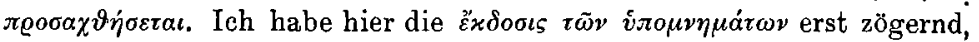
dann. bestimmter auf Editio actionis bezogen, sehe mich aber durch unsern Papyrus eines Besseren belehrt. Es ist Ausfolgung einer Abschrift des Verhandlungsprotokolls, der acta confecta; auf diese ge-

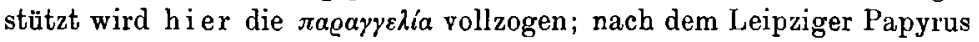

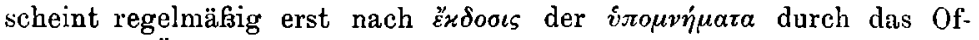
fizium die Überleitung des Verfahrens an den Judex pedaneus erfolgt zu sein. Die Vermutungen für das Ordinarverfahren, die man daran knüpfen kann, liegen nahe genug.

Eine griechische Übersetzung einer lateinisch gefaft gewesencn nmanumissic inter amicos" enthält 1205: sie ist chirographarisch gefafit und sagt in l. 5: $\mu \varepsilon \tau \alpha \xi \dot{v}$ qi $\lambda \omega \nu \hat{\eta} \lambda \varepsilon v \vartheta \varepsilon[\varrho \omega ́ \sigma \alpha \mu \varepsilon \nu$. Dafs die Manumittenton

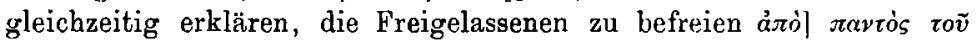

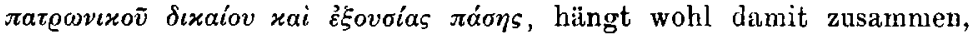

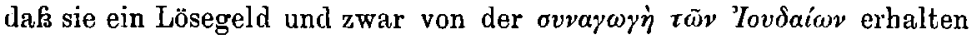
haben; die Freigelassenen sind jedenfalls Juden, die in Oxyrhynchos keineswegs selten waren. Vgl. übrigens auch C. J. 6, 4, 3 pr., worauf auch Lewald hinweist.

1206 ist ein üherraschendes Parallelstück zu P. Lips. 28, eine Adoptio per tabellionem facta v. J. 335, mit den wesentlichen Eigentümlichkeiten der Leipziger Parallelurkunde; nur handelt es sich im Oxyrhynchostext nicht wie im Leipziger um eigentliche Arrogation eines homo sui iuris, sondern um Datio in adoptionem eines Hauskindes; auferdem enthält der erstere den Zusatz, daß der Adoptierte nicht verstofen noch in die Sklaverei soll verkauft werden dürfen. Der Papyrus im Zusammenhalt mit jenem zeigt, dafs die adoptio per tabellionem keine Seltenheit war und trägt damit wesentlich zur Erklärung der justinianischen Adoptio minus plena bei, welche man schon nach Lips. 28 auf den Einfluß einer Volkssitte zurückführen muste.

Mit einigen kleineren verwaltungsrechtlichen Stücken und einer Anzahl von Briefen schliefst der Band ab, in welchem man gern noch fortgelesen hätte.

II. Endlich veröffentlicht Friedrich Preisigke das dritte und Schlußheft des ersten Bandes seiner Ausgabe der Papyri der Straßsburger Universität; es ist mir ein Bedürfnis neuerlich zu sagen, wie sehr ich 
diese Publikation eines in praktischen Verwaltungsdienst stehenden Gelehrten schätze, der sich in kurzer Zeit den nicht mehr zu erschütternden Ruf eines der gründlichsten Papyruskenner erworhen hat; die Straßburger Publikation nimmt durch die Verläßlichkeit ibrer Lesungen und die eminente Sorgfalt und Gründlichkeit ihrer Kommentare, außerdem auch durch praktische und schöne Ausstattung eine feste Stelle in den vordersten Reihen der Papyrusausgaben ein. Im vorliegenden Heft sind zunächst eine Anzahl von Ergänzungen und Fmendationen zu den früheren Texten mitgeteilt; ihren Schluf der sorgfältig gegliederte Wort- und Sachindex. Ls ist begreiflich, dafs die größten und wichtigsten Urkunden der Sammlung schon in den früheren Heften mitgeteilt worden sind; darum gibt das jetzt erschienene mehr eine Nachlese kleinerer Stücke, hauptsächlich die Verwaltung betreffend. Eines, Nr. 56, das sich anscheinend auf einen Rechtsstreit über ein Haus bezieht, übrigens nach der Schriftprobe wohl nur das Protokoll eines niederen Beamten enthält, ist zu sehr zerstört, um näher erörtert werden zu können; zum Text bemerke ich nur, dafo in Fragment B lin. 21/2 natürlich nicht ergänzt werden darf

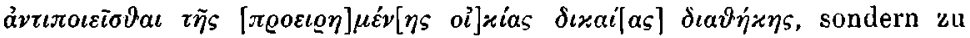

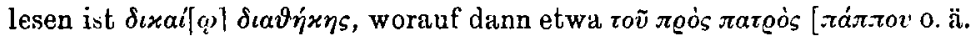
Die falsche adjektivische Ergänzung des Wortrestes $\delta$ ¿xal ist iibrigens in den Editionen häufig; ich will keine Parallelfälle zitieren. Nr. 79 ist die authentische Publikation des Sklavenkaufs v. J. 16/15 v. C., auf den Kübler in dieser Ztschr. 32 als eine für den Begriff' der ėrap í-manus injectio entscheidende Urkunde lingewiesen hat.

Im Leipziger Dekanatsprogranm 1912 habe ich selbst zwei Urkunden publiziert; ein gerichtliches Verhandlungsprotokoll in griechischer Sprache aus dem 4.-5. Jhd, der Berliner Sammlung gehürigr, und eine lateinische Emanzipationsurkunde der Leipziger Sammlung aus Oxyrhynchos, welche, der nachantoninischen Zeit angehörig, doch den klassischen Stil der Mancipatio fiduciae causa in einer für Zeit und Ort höchst merkwürdigen Reinheit (mit nur geringen Unregelmäß̈igkeiten) erkennen läßt. Leider ist man bei der Edition auf konjekturale Restitution der sehr beträchtlichen luücken des Textes angewiesen; zu der in der Ausgabe vorgenommenen Ergïinzung ist mir eine Anzahl von Vorschlägen besonders von Lenel, Moriaud, Eger zugegangen, deren Mitteilung wir jedoch zu meincm Bedauern wegen Raummangels an dieser Stelle nicht möglich ist.

Gries-Bozen November 1912.

Mitteis. 